\title{
Engineering Crowd Interaction within Smart Environments
}

\author{
M.D. Harrison \\ School of Computing Science, \\ Newcastle University, Claremont \\ Tower, \\ Newcastle upon Tyne, NE1 7RU, UK \\ +441912228218 \\ michael.harrison@ncl.ac.uk \\ Mieke Massink \\ Istituto di Scienza e Tecnologie Istituto di Scienza e Tecnologie \\ dell'Informazione 'A. Faedo', CNR dell'Informazione 'A. Faedo', CNR \\ Area della Ricerca, Via G. Moruzzi 1, Area della Ricerca, Via G. Moruzzi 1, \\ Pisa, Italy \\ +390503152981 \\ Pisa, Italy \\ +39050 3152982 \\ mieke.massink@isti.cnr.it \\ diego.latella@isti.cnr.it
}

\begin{abstract}
Smart environments (e.g., airports, hospitals, stadiums, and other physical spaces using ubiquitous computing to empower many mobile people) provide novel challenges for usability engineers. Firstly, interaction can be implicit and therefore unintentional on the part of its users. Secondly, the impact of a smart environment can influence the collective or crowd behavior of those immersed within it. These challenges lead to requirements for complementary analyses which must be combined with the more typical focus on the interaction between user and device. The paper explores a family of stochastic models aimed at analyzing these features with a particular focus on crowd interaction.
\end{abstract}

\section{Categories and Subject Descriptors}

D.2.4 [Software Engineering]: Software/Program Verification statistical methods. D.4.8 [Performance]: Stochastic analysis user interfaces. H.5.2 [Information Interfaces and

Presentation]: User Interfaces - theory and methods.

\section{General Terms}

Design, Reliability, Human Factors, Verification

\section{Keywords}

Formal Methods, Usability analysis, Performance Evaluation Process Algebra, Dynamic Signage Systems.

\section{INTRODUCTION}

Smart environments, that is ubiquitous computing systems designed to support users, travelers, patients, shoppers in physical environments, are an important area for future growth of computer based systems. These systems, embedded within physical spatial settings, combine the use of sensors, public displays and hand held mobile devices to create an experience of place and to provide information and services relevant to the environment. Envisaged environments include airports, shopping complexes, hospitals, stadiums, civic buildings. While these systems pose many familiar usability challenges relating to interaction with the displays and the mobile devices, for example

Permission to make digital or hard copies of all or part of this work for personal or classroom use is granted without fee provided that copies are not made or distributed for profit or commercial advantage and that copies bear this notice and the full citation on the first page. To copy otherwise, or republish, to post on servers or to redistribute to lists, requires prior specific permission and/or a fee.

EICS'09, July15-17, 2009, Pittsburgh, Pennsylvania, USA.

Copyright 2009 ACM 978-1-60558-600-7/09/07...\$5.00. how to organize the displays and make actions available to resource users [13], there are additional challenges.

These environments may be designed to provide a number of solutions for their stakeholders, for example dealing with regulation of waiting times and supporting the user "journey". A well designed environment can improve experience by providing the right information when it is needed. It can be used to improve crowd-system interaction - how long people have to wait for their information, how people are affected by others. Formal models of the whole system, including the users within the system, can be used to "calculate" some of the effects of a proposed design before deployment. Novel usability aspects in relation to these systems include: (1) the effect of implicit or incidental user interaction; (2) the mutual influences of collective user interaction.

These properties of the whole system are complex to analyze. How to perform this analysis has so far been neglected. Because these systems involve many elements, including many users, and some of the crowd effects are stochastic in nature, new modeling techniques are required. The aim of the paper is to use a stochastic modeling technique to indicate how the effect of a particular smart environment design can impact the collective behavior of users of the environment. PEPA [8] and the analysis techniques associated with PEPA provide a scalable approach. The paper explores analysis techniques based on PEPA [8] including the use of stochastic model checking and flow analyses to explore both these aspects of the system, but with a particular emphasis on crowd effects.

This is the first time a fluid flow analysis based on stochastic process algebras has been used for the analysis of large populations in smart environments. This approach can handle models incorporating large populations of users, and environments involving a realistic number of spaces in a time efficient manner. This makes the technique described superior to traditional simulation and model checking techniques. It can be used in the early design stages of a smart environment where the developers can make assumptions about predicted volumes of people within the environment.

The paper uses an example, inspired by a guidance system, to explore the feasibility of the approach. The system is designed to help people locate a space within a building. In the example, considered in detail here, a small number of spaces is considered for reasons of presentation. A larger example is considered in the technical report [7]. In this example the searched for location could be a person's office, or a seat in a building or the exit in a stadium or theatre, or it might be used to evacuate these buildings. The system assumes that each "visitor" (the term visitor will be used even though this may not be appropriate for the application) 
has some form of electronic tag, for example an RFID on a visitor pass. Alternatively the visitor could use their Bluetooth enabled mobile phone. It is assumed that sensors within the environment will recognize the location and identify the user.

This example system forms the basis of the analysis. It is realistic and representative of a large class of systems. Similar analysis could be done for a broader range of applications by adapting the generic models to specific applications. The physical space contains public displays that provide directional information. Each display contains a relatively small number of "slots" (depending on the real estate of the display and other usability considerations) each indicating the direction for a particular destination. Hence if the ticket, held by the user, is for block D and row 13 then this destination would be indicated along with the direction indicating the next space they should go to get to the seat.

\section{RELATED WORK}

Systematic techniques for the analysis of usability tend to focus mainly on the interaction between the individual user and the device: classical techniques such as cognitive walkthrough [12] focus on goals and actions; heuristic evaluation focuses on how well the device supports given design heuristics [11]. Analogous techniques have been formalized with the aim of making them systematic, tool supported and applicable by people who are not expert in human computer interaction, see for example $[2,5]$. In smart environments, users are immersed within the system and therefore some properties of the user must be captured in the model. The experience that is created by these systems becomes more important as a result. How experience is captured and analyzed in these formal models is an important issue. Some properties can be captured as properties of the systems that are derived from specific user evaluations [6]. For instance, allaying the anxiety of users within the environment, achieved by ensuring that users have the information they need when and where they want it. When they sit in a seat in the main airport hall, for example, they are able to see the display of up-to-date flight information that is relevant to them. These are properties that can potentially be checked of the system. Other relevant properties concern timeliness of visitors arriving at destination, congestion and reduced capacity. Moreover, the models can in principle accommodate certain kinds of user behavior such as users with reduced capabilities of movement or user making errors in following guidance instructions.

\section{MODELING THE INTERACTION}

The focus of the smart environment model is the interaction between a visitor and the display in a location, and action that is taken as a result. Displays are linked to logical spaces. Each display has a finite number of slots, and each slot may show a direction linked to a destination. The display only shows directions for destinations relevant to the occupants of the space. Therefore not all the slots may be used if there are fewer destinations required amongst the assembled visitors. Alternatively all the slots may be used and assembled visitors may have to wait. The display cannot be read by everybody in the space, it has specified capacity. While this description suggests an OR like resource model, the specification produced also captures the architecture of the smart environment and can be used to explore a range of relevant usability questions.
These assumptions lead to a number of issues associated with the system, in addition to those relating to the usability and format of the display. The following properties capture a sample of the analysis of variants of the system.

P1: the system guarantees that relevant information is always available eventually, ensuring that the visitor eventually reaches a destination.

P2: The system guarantees that the visitor always gets closer to the destination if following the sign.

P3: There is a probability $p$, depending on visitor volumes over time, that the visitor will have to wait for information relevant to them on the display.

P4: The average time taken for visitors to reach their destination is $t$, assuming different volumes of visitors over time.

These properties relate to the user's experience and interaction with the system. This is particularly true if the experience of the system is concerned with (1) waiting for the display to be relevant to them; (2) the total time taken to reach the destination. One might expect, as discussed in [6], that properties such as these will be elicited from typical visitors within the system.

A model is required that reflects the structure of the proposed system and also enables the analysis of behaviors and proposed crowd flows.

\subsection{PEPA Modeling}

PEPA components reflect the behavior of relevant parts of the system in terms of activities that capture the actions that the components perform. A component may itself be composed of components. A PEPA activity consists of a pair (action type, rate) in which action type denotes the type of the action, while rate characterizes the negative exponential distribution of the activity duration. A positive real-valued random variable $\mathrm{X}$ is exponentially distributed with rate $r$ if the probability of $X$ being at most $t$, i.e. $\operatorname{Prob}\{X \leq t\}$, is $1-e^{r . t}$ if $t \geq 0$ and is 0 otherwise, where $t$ is a real number. The expected value of $X$ is $1 / r$.

Exponentially distributed random variables are more tractable because they are memoryless, i.e. $\operatorname{Prob}\left\{X>t+t^{\prime} \mid X>t^{\prime}\right\}=\operatorname{Prob}\{X$ $>t\}$ for $t, t^{\prime} \geq 0$ and are widely used in the modeling of the dependability and performance of real systems. Compositions of exponential distributions can be used for the approximation of any non-negative distribution.

PEPA is a simple process algebra. Its syntax is:

$$
\mathrm{P}::=\left.(\mathrm{a}, \mathrm{r}) \cdot \mathrm{P}|\mathrm{P}+\mathrm{P}| \mathrm{P}\right|_{\{\mathrm{L}\}} \mathrm{P} \mid \mathrm{A}
$$

Here ( $a, r) . P$ describes a component $P$ that is guarded by the activity $(a, r)$ where a is an action type and $r$ is the rate. $P+Q$ is a choice between the two processes $\mathrm{P}$ and $\mathrm{Q}$. The cooperation operator $\left.\mathrm{P}\right|_{\{\mathrm{L}\}} \mathrm{Q}$ defines the set of action types $\mathrm{L}$ on which components $\mathrm{P}$ and $\mathrm{Q}$ synchronize. Both components proceed independently with any activity not occurring in L. The expected duration of a co-operation of activities $a$ in $L$ is a function of the expected durations of the corresponding activities in the components, typically the longest one. The behavior of process variable $A$ is that of $P$, provided that a defining equation $A=P$ is available for A.

If the set $L$ is empty $P||_{\{L\}} Q$ is written: $P \mid Q$. Multiple (n) copies of $\mathrm{P}$ co-operating in parallel with $\mathrm{m}$ parallel copies of $\mathrm{Q}$ is 
written: $\mathrm{P}[\mathrm{n}] \mid \|_{\{\mathrm{L}\}} \mathrm{Q}[\mathrm{m}]$. The details of the formal semantics are described in [8].

Stochastic models based on PEPA can be time consuming to produce. This is a problem if the aim is to compare results in relation to different routing schedules, different groups of visitors or even varying layouts of the buildings. The PEPA process algebra is simple, involving few basic operators, and it provides versatility in terms of the different automatic analysis techniques that can be applied to the same specification. However its very simplicity means that specifications can become lengthy. It is necessary to encode many aspects of the modeled system using processes and action names.

A program was written therefore to automatically synthesize PEPA models, given domain specific input such as the layout of the building, routing information, grouping of visitors and their final destination. The PEPA specifications for the guidance system were made sufficiently modular and each component as general as possible, to enable the appropriate instantiation. The synthesizing program is described in [7].

The rate assumptions used were: $\mathrm{s}=10$ (visitor takes 6 secs to see display); $\mathrm{a}=1$ (visitor takes $1 \mathrm{~min}$ to request information); $\mathrm{r}=2$ (slot takes 30 secs to display information); $\mathrm{rr}=10$ (visitor takes 6 secs to read information already shown); $\mathrm{f}=0.2$ (information remains 5 minutes on the display). The exact parameters can be tuned for the particular case at hand.

\subsection{PEPA analysis}

PEPA models can be used as a basis for stochastic modelchecking, and stochastic simulation [3]. The current example has many instantiations of the same process. A new technique is particularly appropriate that involves translating the PEPA model into a set of Ordinary Differential Equations (ODEs). The innovation is to use the number of visitors that are in a particular state over time, instead of representing the state itself for each individual visitor. This approach considerably reduces the state space explosion problem. A description of the approach can be found in [9]. The results are compared with stochastic simulation in [7] where it is shown that the approach provides a cost effective and robust alternative to simulation for the examples illustrated.

\subsection{The PEPA model of the guidance system}

As illustration an example is used based on Figure 1. Slots and places are instantiated for each particular location. The display consists of a number of slots. The slot manager and the arbitrator in each location ensure that requested information is displayed and that no two slots show the same information. Groups of visitors are defined where each visitor in the group has the same starting location and final destination. For example, a visitor starting in location A and heading for location D first tries to get a place in location A where it is possible to see the display (lasd is the action of trying to acquire a place in location A). Once a place has been acquired the individual engages in action laee to find out where to go next. The request is engaged as soon as there is an available display slot that displays the information. When the information is displayed the visitor releases the place in location A (action lasu) and receives the information (i.e. any of the matching destinations in the process VisEdRec). The visitor then proceeds to the indicated next location (e.g. VisEdtoLb means that the visitor with final destination D must first proceed to location
B). The arrival at the final destination is modeled by the process that remains in the state VisEdArrived forever.

The visitor VisEytoLx, currently at location $\mathrm{x}$, wishing to reach destination $\mathrm{y}$, is defined in PEPA as:

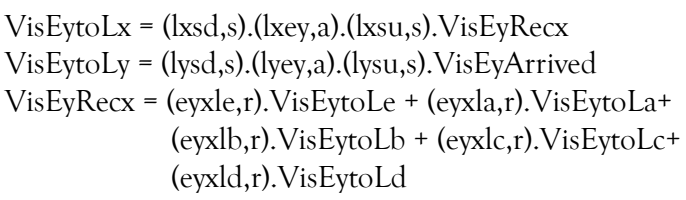

The visitor that has arrived at location y is defined as:

VisEyArrived $=($ nop,a). VisEyArrived

It takes on average $1 / \mathrm{s}$, i.e. 6 seconds (see previous section) for a visitor to find a place to see the display. Different groups of visitors are modeled by combining groups of visitors. nop is an action not engaged.

Vis $=($ nop, $\mathrm{v} 0) \cdot$ VisEetoLa $+($ nop, $v 1) \cdot$ VisEdtoLa

The rates $\mathrm{v} 0$ and $\mathrm{v} 1$ model the arrival rates of individuals of each kind. Vis will be combined with other processes with multiple instances.

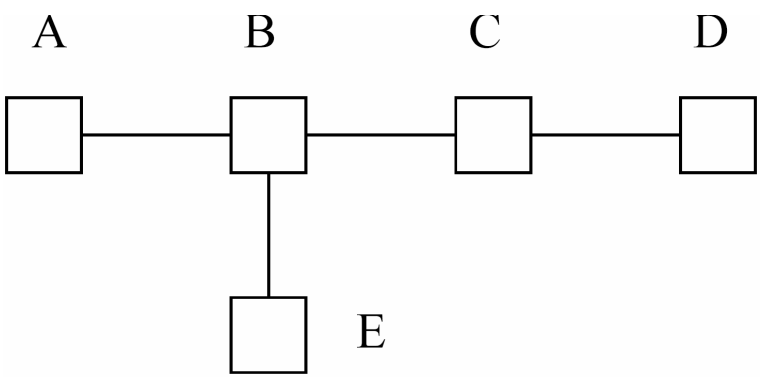

Figure 1. Layout of the building used in the illustration

Each location uses an arbitrator process to display directions to destinations depending on requests from visitors. The arbitrator ensures that a slot in a location does not show information that is already displayed by another slot in the same location. Process ArbLaEe starts with an empty display and waits for a request for information from a visitor. If such a request arrives (laee) it sets up a slot to display the required information by sending a request to the Slot Manager process (slaee). Further requests for the same information are then handled directly by the relevant slot. Requests for routing information for different destinations are still handled by the arbitrator which will start up new slots via the Slot Manager as long as there are empty slots available. Slots become free and the arbitrator is informed via the Slot manager (sfreelae) and returns to its initial state. The activities internal to arbitrators and slots are considered to be relatively fast, which explains the chosen fixed rate 100 .

ArbLxEy = (lxey,a).(slxey,100.0). $($ sfreelxy,100.0).ArbLxEy

If a location is only a final destination the arbitrator does not need to set up a display slot for an individual:

ArbLdEd $=($ lded, $\mathrm{a}) \cdot$ ArbLdEd

The Slot Manager (SmanLxy) initializes and releases slots. It receives a request from the arbitrator (slxey) of the location to setup a slot that displays routing information to a particular destination. The request is followed by an initialization message from the Slot Manager to a free slot (sslxey). That the slot is now free is notified to the Slot Manager (freelxy) and then forwarded to the arbitrator of the location that keeps track of free slots and the 
information on display (sfreelxy). The process for a slot manager at location $\mathrm{x}$ dealing with requests for destination $\mathrm{y}$ is:

$$
\begin{aligned}
\text { SmanLxy }= & (\text { slxey, 100.0). (sslxey, 100.0). } \\
& (\text { freelxy,f).(sfreelxy, 100.0).SmanLxy }
\end{aligned}
$$

A display slot for location A can be set to display direction information for a particular destination after a message from the Slot Manager, for destination D (sslaed) and destination E (sslaee). The process handles requests for destination $\mathrm{D}$ after the slot has been set up for that destination. When a slot already displays the directions for a particular destination then the response is much faster, given by rate rr. The information can be removed from the display by an autonomous action of the slot called freelxy labeled by the relevant location ( $\mathrm{x}$ ) and destination (y) for which it was displaying information.

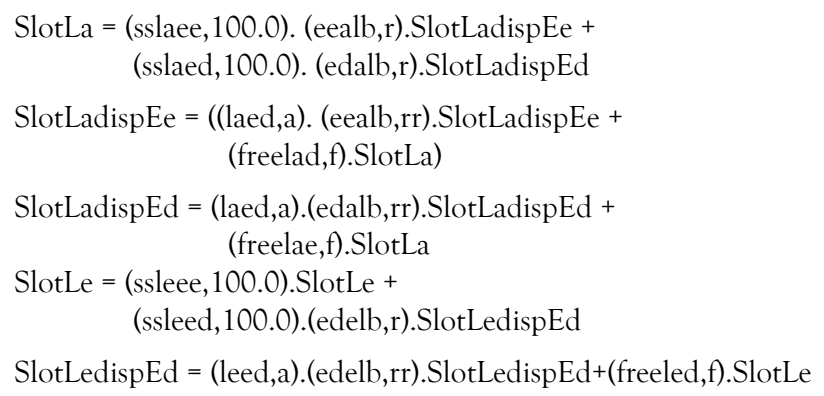

Whether it is possible for a visitor to see the display (i.e., a place is free) is modeled by:

PlaceFreeLa $=($ lasd,s).(lasu,s).PlaceFreeLa

For a system with 60 visitors, 5 locations with 10 places per location from where the display is visible, and displays with 4 slots, the composed system can be specified as follows:

(Vis[60] | Slotmanagers) | | (Slots | Arbitrators | Places)

where for each final destination $y$ :

Slotmanagers $=$ SmanLay[1] $|\ldots|$ SmanLey[1]

Slots $=$ SlotLa[4] | ... SlotLe[4]

Arbitrators $=$ ArbLaEy[1] $|\ldots|$ ArbLeEy[1]

Places $=$ PlaceFreeLa[10] $|\ldots|$ PlaceFreeLe[10]

$\mathrm{L}$ is the set of all the actions that these processes have in common. 90 ordinary differential equations are generated in this case.

\section{ANALYSIS}

A number of factors influence the analysis of the properties, P1P4. For example, information about how quickly a visitor will get to their destination will depend on typical volumes of people and this depends on the system's purpose. For example, providing guidance to people finding their seat in an auditorium will involve a relatively short period over which people arrive (perhaps an hour) and a hard deadline for reaching seats. In such a system people will arrive through a few entry points and will need to reach a large number of destinations. The usability concerns here will relate to how clear the displays are (whether they can be interpreted correctly), the routes, the flows and the waiting times.

PRISM [10] (a stochastic model checker) was used to verify properties expressed in the Continuous Stochastic Logic (CSL) based on P1 and P2 for a much reduced system with 4 visitors. Questions addressed by this analysis included properties that have as a main purpose to verify several correctness issues rather than issues regarding the quantitative effect of the presence of many visitors. Some examples are:

What is the probability that a visitor starting at A, heading for location $D$ currently in $C$, goes back to location B?

$P=?$ [true $U($ Vis_STATE $=$ VisEdtoLb) $\{$ Vis_STATE $=$ VisEdtoLc $\}]$

This property can be used to check that visitors are never getting further away from their final destination (P2). The specification defined in the paper resulted in probability 0 . Another example is:

What is the probability that visitors for exit $E$ and exit $D$ arrive at their respective destinations within 5 time units? $P=$ ? true $\mathrm{U}_{<=5}$

(Visea_STATE= VisEeArrived\&Visda_STATE=VisEdArrived)]. The result of analyzing this property (relevant to P1) subject to assumptions beyond current scope is a probability of 0.017690 . Stochastic model checking of systems with a large number of visitors creates a state-space problem, hence the exploration of the scalable Fluid Flow (ODE) technique.

The PEPA workbench [4] offers various methods for the analysis of collective behavior including stochastic simulation and ODE analysis. The PEPA workbench translates the model automatically into a set of ODEs and uses an adaptive step-size $5^{\text {th }}$ order Dormand Prince ODE solver to analyze the system [9].

While simulations can always be done for the specifications requiring a large number of independent replications, they can be time-consuming if a certain level of accuracy is required. The reliability of the results obtained with ODE can easily (and quickly) be checked by comparing the results with a "limited" simulation with only a few independent executions. If there is reasonable correspondence then the ODE results can be used. Simulation however produces more irregular results when few independent executions are used. The reason for occasional noncorrespondence of the results is still a topic of research.

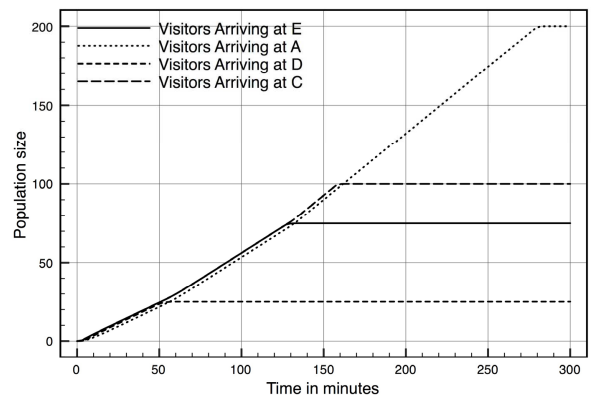

Figure 2. Number of visitors arriving at destination E, A, D and $C$ : the ODE analysis

The model assumed a group of 25 visitors starting in location A and heading for $\mathrm{D}$ with arrival rate $0.0625,75$ starting at $\mathrm{C}$ heading for $\mathrm{E}$ with arrival rate $0.1875,100$ starting at $\mathrm{A}$ heading towards $\mathrm{C}$ with rate 0.25 and 200 starting at $\mathrm{D}$ and heading for $\mathrm{A}$ with rate 0.5 . Taking the latter as an example, this means that at location D 200 visitors are waiting to enter, each one taking on average 2 minutes to do so.

The first ODE analysis (Figure 2) shows the number of visitors of the different groups that reached their destination over time (in minutes). After 50 minutes all 25 visitors with final destination D 
have arrived. This scenario could be about evacuation but it could also relate to a hospital where in the morning people arrive for a blood-test within a particular time interval and where after 50 minutes on average all patients have received their treatment in room $\mathrm{D}$ and gone home.

It is also possible to analyze the number of visitors that are occupying places in the various locations thus providing insight into potential congestion. The data make it possible to compare alternative routes for groups of visitors through the building, as defined by the smart environment. Analysis indicates that the starting locations for the visitors (A and D) are fully occupied for some time. Location D is occupied for almost the whole period until all visitors have arrived at their final destination location. These results are not surprising given the destinations of the visitors.

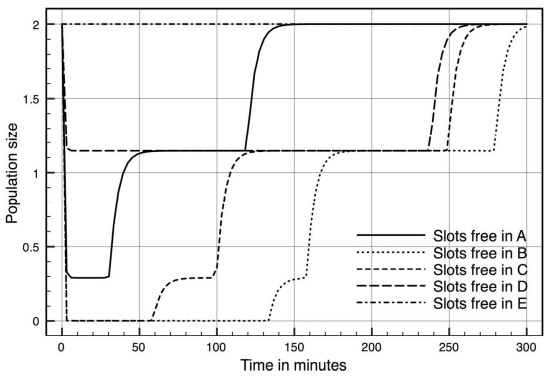

Figure 3 Number of empty slots over time (ODE Analysis)

The second analysis (Figure 3) shows how many slots are empty over time. They give an impression of the results of a simulation based on an unbounded number of independent executions. Although visitors are heading for 4 different destinations at most two slots are occupied at any time as was specified in the model. In location $\mathrm{D}$ at most one slot is used. In fact, only visitors coming in at $\mathrm{D}$ and heading for location $\mathrm{A}$ (via $\mathrm{C}$ ) request information in location D.

\section{DISCUSSION AND FUTURE WORK}

This paper briefly explores the analysis of a realistic class of smart environments using stochastic model checking and fluid flow analysis. A more detailed description can be found in a technical report [7]. Several variants of a dynamic signage system were explored. While the example is not overcomplicated it captures many of the interesting features of smart environments including the characteristics of implicit interaction and the mutual influence of people flowing through the environment. Although the models are quite general, they can be tuned to particular applications quite easily. The main purpose in this paper is to show the general feasibility of the approach for a stylized example which nevertheless contains many of the typical aspects of smart environments shared by many people.

Using these abstractions it has been shown that PEPA models with many visitors, a realistic number of rooms (current maximum analyzed 26 rooms) with a high capacity, can be analyzed efficiently with acceptable computation costs. Figure 4 shows the number of visitors arriving from locations $\mathrm{a}, \mathrm{f}, \mathrm{w}$ and $\mathrm{z}$ at location g, see layout in Figure 5.

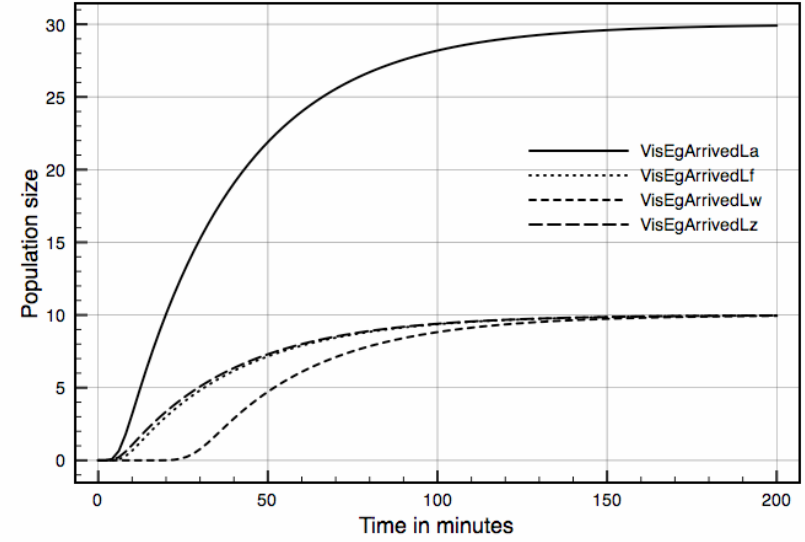

Figure 4 Number of visitors from different locations arriving at location $G$ over time.

These promising results provide a first indication of how formal methods may be used to explore a space of different designs of smart environments before their deployment with relatively little effort, focusing on issues of crowd system interaction. Different designs based on the size of spaces, numbers of slots and assuming different routes may be rapidly constructed and analyzed. Different traffic assumptions can be embedded in the rate values and number of instantiations of processes. There are a number of important and interesting technical aspects of this problem that move beyond this preliminary study. A key concern is the issue of validation of these models. Some known systems, with many users, have been modeled, strategies have been adopted that are not supported by smart environments (see, for example [1]) and their results have been compared with real observations. A future issue is to develop these empirical analyses in relation to the proposed smart environments. These results bring a distinctive new perspective to usability in smart environments and establish a new agenda for their analysis.

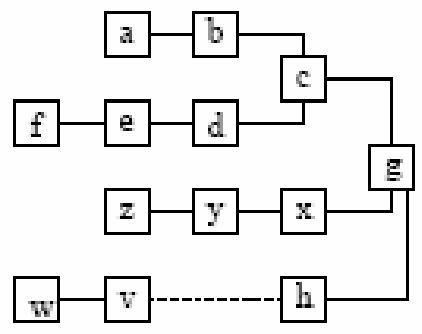

Figure 5 A more substantial layout

There are also usability issues to address more effectively within the modeling approach. A model of the human is embedded in the model of the system. Instead of the more elaborate or prescriptive models developed by cognitive modelers or task modelers, these models make minimal assumptions about the cognitive capabilities or domain knowledge of the user. In the case of the models described here the user always follows instructions correctly, and will remember them perfectly when they are in an area that does not contain a display. It is clear that these assumptions can be relaxed. An interesting question is how they 
should be relaxed in a way that is consistent with general characteristics of human behavior.

The analysis also raises interesting questions about how generalizable these results are to a range of smart environments. While further work remains to fully analyze the existing environment, further developments relating to the characterization of smart environment models raise additional challenges. Firstly it is to be envisaged that smart environments will also involve direct engagement between user and the environment. The more usual techniques of usability analysis will also be required in the analysis of a proposed design. The model of the user must therefore capture these explicit engagements in the immersed user as well as the implicit and collective. Secondly, smart environments will not as a rule have the regular characteristics of the systems described here. Airports and hospitals will require information and configurations that are particular to the spaces they are located in. In these cases there are relatively few spaces but the processes described within them are different, requiring tuning and extension of the models presented so far preserving though their basic structure and approach.

\section{ACKNOWLEDGMENTS}

We thank Stephen Gilmore for suggestions concerning modeling issues, the EU Resist network of excellence and the Italian CNR project XXL.

\section{REFERENCES}

[1] Au-Yeung, S.W.M., Harrison, P.G. and Knottenbelt, W.J. 2006. A queueing network model of patient flow in an accident and emergency department. In Proceedings $20^{\text {th }}$ Annual European and Simulation Modelling Conference, 60-67.

[2] Campos, J.C. and Harrison, M.D. 2008. Systematic analysis of control panel interfaces using formal tools. In N. Graham and P. Palanque, editors, Interactive systems: Design, Specification and Verification, DSVIS'08, Springer Lecture Notes in Computer Science, 5136 72-85. Springer-Verlag,

[3] Gillespie, D.T. 1977. Exact stochastic simulation of coupled chemical reactions. The Journal of Physical Chemistry, 81(25):2340-2361.

[4] Gilmore, S. and Hillston, J. 1994. The PEPA workbench: a tool to support a process algebra-based approach to performance modelling. In Proceedings of the Seventh International Conference on Modelling Techniques and
Tools for Computer Performance Evaluation, Springer Lecture Notes in Computer Science 794, 353-368. SpringerVerlag.

[5] Gow, J. and Thimbleby, H.W. 2004. MAUI: An interface design tool based on matrix algebra. In R. J. K. Jacob, Q. Limbourg, and J. Vanderdonckt, editors, Computer Aided Design of User Interfaces IV, CADUI' 2004, 81-94.

[6] Harrison, M.D., Kray, C., Sun, Z and Zhang, H. 2008. Factoring user experience in the design of ambient and mobile systems. In Gulliksen, J.; Harning, M.B.; Palanque, P.; Veer, G.C. van der; Wesson, J. (Eds.) Engineering Interactive Systems Springer Lecture Notes in Computer Science. Vol. 4940. 243-259.

[7] Harrison M., Massink M., Latella D. Engineering human flows in smart environments using formal techniques - Full version. 2009. <http://puma.isti.cnr.it/ metadata.php? idcode $=2009-$ TR-014\&idcol $=1 \&$ idauth $=1 \&$ langver $=i t>$. Technical report CNR-ISTI 2009-TR-014.

[8] Hillston, J.A.. 1996. A compositional approach to performance modelling. C.U.P.

[9] Hillston, J. 2005. Fluid flow approximation of PEPA models. In Proceedings of QEST'05, 33-43. IEEE Computer Society.

[10] Kwiatkowska, M., Norman, G. and Parker, D. 2002. PRISM: Probabilistic symbolic model checker. In T. Field, P.G. Harrison, J. Bradley, and U. Harder, editors, Computer Performance Evaluation: Modelling Techniques and Tools Proceedings 12th International Conference, TOOLS 2002, Springer Lecture Notes in Computer Science, 2324, page 200. Springer-Verlag.

[11] Nielsen J. 1992. Finding usability problems through heuristic evaluation. In: Proc. of ACM CHI'92 Conference on Human Factors in Computing Systems. New York. ACM 249-256.

[12] Polson, P.G., Lewis, C., Rieman, J. and Wharton, C. 1992. Cognitive walkthroughs: a method for theory-based evaluation of user interfaces, International Journal of ManMachine Studies, Volume 36(5).

[13] Wright, P.C., Fields, R.E. and Harrison, M.D. 2000. Analyzing human-computer interaction as distributed cognition: the resources model. Human Computer Interaction, 15(1):1-42. 